\title{
Respiratory infections are temporally associated with initiation of type 1 diabetes autoimmunity: the TEDDY study
}

\author{
Maria Lönnrot ${ }^{1,2}$ • Kristian F. Lynch ${ }^{3}$ - Helena Elding Larsson ${ }^{4,5}$ • Åke Lernmark ${ }^{5,6}$ • \\ Marian J. Rewers ${ }^{7}$ - Carina Törn ${ }^{4,5}$ • Brant R. Burkhardt ${ }^{3}$. Thomas Briese ${ }^{8,9}$. \\ William A. Hagopian ${ }^{10}$ • Jin-Xiong She ${ }^{11}$. Olli G. Simell ${ }^{12,13}$ • Jorma Toppari ${ }^{14,15}$. \\ Anette-G. Ziegler ${ }^{16,17}$ • Beena Akolkar ${ }^{18}$ - Jeffrey P. Krischer ${ }^{3}$ Heikki Hyöty ${ }^{2,19}$ • on \\ behalf of the TEDDY Study Group
}

Received: 18 January 2017 / Accepted: 5 June 2017 / Published online: 2 August 2017

(C) Springer-Verlag GmbH Germany 2017

\begin{abstract}
Aims/hypothesis Respiratory infections and onset of islet autoimmunity are reported to correlate positively in two small prospective studies. The Environmental Determinants of Diabetes in the Young (TEDDY) study is the largest prospective international cohort study on the environmental determinants of type 1 diabetes that regularly monitors both clinical infections and islet autoantibodies. The aim was to confirm the influence of reported respiratory infections and to further
\end{abstract}

Electronic supplementary material The online version of this article (doi:10.1007/s00125-017-4365-5) contains peer-reviewed but unedited supplementary material, which is available to authorised users.

Maria Lönnrot

maria.lonnrot@uta.fi

1 Department of Dermatology, Tampere University Hospital, Teiskontie 35, 33521 Tampere, Finland

2 Faculty of Medicine and Life Sciences, University of Tampere, Tampere, Finland

3 Health Informatics Institute, Morsani College of Medicine, University of South Florida, Tampa, FL, USA

4 Department of Clinical Sciences Malmö, Clinical Research Centre (CRC), Lund University, Malmö, Sweden

5 Skåne University Hospital (SUS), Malmo, Sweden

6 Department of Clinical Sciences Malmö, Lund University Clinical Research Centre (CRC), Malmö, Sweden

7 Barbara Davis Center for Childhood Diabetes, University of Colorado, Aurora, CO, USA

8 Center for Infection and Immunity, Mailman School of Public Health, Columbia University, New York, NY, USA

characterise the temporal relationship with autoantibody seroconversion.

Methods During the years 2004-2009, 8676 newborn babies with HLA genotypes conferring an increased risk of type 1 diabetes were enrolled at 3 months of age to participate in a 15 year follow-up. In the present study, the association between parent-reported respiratory infections and islet autoantibodies at 3 month intervals up to 4 years of age was evaluated in 7869 children. Time-dependent proportional hazard Columbia University, New York, NY, USA

10 Pacific Northwest Diabetes Research Institute, Seattle, WA, USA

11 Center for Biotechnology and Genomic Medicine, Medical College of Georgia, Augusta University, Augusta, GA, USA

12 Research Centre of Applied and Preventive Cardiovascular Medicine, Faculty of Medicine, University of Turku, Turku, Finland

13 Department of Paediatrics and Adolescent Medicine, Faculty of Medicine, University of Turku, Turku, Finland

14 Department of Physiology, Institute of Biomedicine, University of Turku, Turku, Finland

15 Department of Paediatrics, Turku University Hospital, Turku, Finland

16 Forschergruppe Diabetes e.V, Neuherberg, Germany

17 Institute of Diabetes Research, Helmholtz Zentrum München, Munich, Germany

18 National Institute of Diabetes \& Digestive \& Kidney Diseases, Bethesda, MD, USA

19 Fimlab Laboratories, Pirkanmaa Hospital District, Tampere, Finland 
models were used to assess how the timing of respiratory infections related to persistent confirmed islet autoimmunity, defined as autoantibody positivity against insulin, GAD and/ or insulinoma antigen-2, concordant at two reference laboratories on two or more consecutive visits.

Results In total, 87,327 parent-reported respiratory infectious episodes were recorded while the children were under study surveillance for islet autoimmunity, and 454 children seroconverted. The number of respiratory infections occurring in a 9 month period was associated with the subsequent risk of autoimmunity $(p<0.001)$. For each $1 /$ year rate increase in infections, the hazard of islet autoimmunity increased by $5.6 \%$ (95\% CI 2.5\%, 8.8\%). The risk association was linked primarily to infections occurring in the winter (HR $1.42[95 \%$ CI $1.16,1.74] ; p<0.001)$. The types of respiratory infection independently associated with autoimmunity were common cold, influenza-like illness, sinusitis, and laryngitis/tracheitis, with HRs $(95 \%$ CI) of 1.38 (1.11, 1.71), 2.37 (1.35, 4.15), $2.63(1.22,5.67)$ and $1.76(1.04,2.98)$, respectively.

Conclusions/interpretation Recent respiratory infections in young children correlate with an increased risk of islet autoimmunity in the TEDDY study. Further studies to identify the potential causative viruses with pathogen-specific assays should focus especially on the 9 month time window leading to autoantibody seroconversion.

Keywords Autoimmunity $\cdot$ Islet autoantibodies $\cdot$ Prospective cohort $\cdot$ Respiratory infections $\cdot$ Type 1 diabetes

$\begin{array}{ll}\text { Abbreviations } \\ \text { FDR } & \text { First-degree relative } \\ \text { GADA } & \text { GAD antibody } \\ \text { IAA } & \text { Insulin autoantibody } \\ \text { IA-2A } & \text { Insulinoma antigen-2 autoantibody } \\ \text { RIE } & \text { Respiratory infectious episode } \\ \text { TEDDY } & \begin{array}{l}\text { The Environmental Determinants of Diabetes in } \\ \text { the Young }\end{array}\end{array}$

\section{Introduction}

Type 1 diabetes results in the destruction of pancreatic beta cells through an autoimmune process, marked by the presence of circulating autoantibodies to pancreatic islet cell antigens. Factors promoting islet autoimmunity are not completely elucidated. Genetic factors have a strong influence, but the steep global increase in type 1 diabetes incidence over the last few decades is likely driven by environmental factors that have yet to be determined.

The role of infections as environmental factors linked to type 1 diabetes is unclear; a positive association between infections and diabetes suggests an autoimmunity-promoting effect [1]. However, infections could also protect against autoimmunity [2]. The mode of action may depend on several factors, like microbe species, anatomical location of infection, host's age and the intensity of beta cell stress induced by the infection. Parent-reported early childhood respiratory infections showed no association with islet autoimmunity in a prospective study in the USA [3], whereas respiratory infections were associated with islet autoimmunity in two small European prospective studies; the environmental triggers for type 1 diabetes study (MIDIA) and a primary dietary intervention study to reduce the risk of islet autoimmunity in children at increased risk for type 1 diabetes (BABYDIET) $[4,5]$. An analysis of statutory health insurance claims data of individuals from Bavaria, Germany, suggested an association between early life respiratory infections and later clinical type 1 diabetes [6]. Common microbes that cause respiratory tract infections include enteroviruses, which have been reported to show an association with an increased risk of type 1 diabetes [7-9] and are often found in the pancreatic islets of individuals with type 1 diabetes $[10,11]$. These findings call for further evaluation of the role of respiratory infections in the pathogenesis of diabetes in both participant-reported studies of infections and microbe-specific studies of infections.

The Environmental Determinants of Diabetes in the Young (TEDDY) study has recruited 8676 newborns for a 15 year follow-up, making it one of the largest observational prospective cohort studies of the pathogenesis of type 1 diabetes, and the largest one systematically monitoring both clinical infections and islet autoantibodies. The collection of data on clinical infections $[12,13]$ enables evaluation of rates, timing and types of respiratory infections over time. The aim of the present study was to evaluate the time-dependent associations of respiratory infections with the onset of islet autoimmunity during the first 4 years of life within the TEDDY cohort.

\section{Methods}

Study participants TEDDY was approved by local institutional review or ethics boards and is monitored by an external evaluation committee. There are six clinical research centres: three in the USA (Colorado, Georgia/Florida and Washington State) and three in Europe (Finland, Germany and Sweden). Children with HLA haplotypes conferring an increased risk of type 1 diabetes were invited to participate from both the general population and those with a first-degree relative (FDR) with type 1 diabetes. Written informed consent was obtained from a parent or primary caregiver separately for genetic screening and then participation in the follow-up. A total of 8676 infants born between 2004 and 2010 were recruited into a prospective follow-up from 3 months to 15 years, with study visits every 3 months until the age of 4 years, and then every 6 months thereafter. A detailed study design has been 
previously published $[12,13]$ and details of the TEDDY Study Group can be found in the electronic supplementary material (ESM).

In the present study, follow-up data during the 3 month visit schedule, i.e. from enrolment up to 48 months of age, were evaluated. Participants with ineligible HLA $(n=118)$, indeterminate autoantibody results $(n=55)$, detectable type 1 diabetes-related autoantibodies at enrolment $(n=10)$, or no follow-up visits after enrolment $(n=624)$ were excluded. Participants missing four or more consecutive visits were considered withdrawn from the present study after the date of their last visit. The cohort is described in Table 1.

Determining islet autoimmunity and type 1 diabetes Blood samples drawn at each clinic visit were analysed for insulin autoantibodies (IAA), GAD autoantibody (GADA) and insulinoma antigen-2 autoantibody (IA-2A) $[12,13]$. Samples were analysed at the Barbara Davis Center for Childhood Diabetes at the University of Colorado Denver (US sera) and the University of Bristol (UK) (European sera). All autoantibody-positive samples and 5\% of negative samples were re-tested by the other reference laboratory. Both laboratories exhibited high sensitivity, specificity and concordance [14]. Concordant samples were deemed confirmed. Persistent islet autoimmunity was defined as confirmed autoantibody positivity on more than two consecutive visits at 3 months apart. Type 1 diabetes was diagnosed according to the ADA criteria [15].

Recording of infections Parents recorded symptoms of illnesses and any subsequent diagnosis in a TEDDY diary book. At each scheduled clinic visit, infections since the previous visit were collected by study nurses, who translated parental reports of children's illnesses into diagnosis codes according to the ICD-10 classification by WHO (www.who.int/ classifications/icd/en/). Infectious disease data processing and categorisation in the TEDDY study has been previously described in detail [16]. In brief, we used a respiratory infectious episode (RIE) approach [16], which reduces the possibility of overestimation of microbial exposure because of multiple symptom and/or diagnosis reports during a single microbial infection.

The RIEs are also categorised by having the presence or absence of the following subcategories of a respiratory infections [16]: bronchitis and lower respiratory tract infections; common cold; conjunctivitis (included as this mostly occurs in children in conjunction with a respiratory tract infection and is caused by the same microbes [17]); enterovirus-like illnesses; infections of the middle ear and mastoid process; influenza-like illnesses; laryngitis and tracheitis; respiratory syncytial virus infection; sinusitis; tonsillitis or streptococcal pharyngitis; other respiratory tract infections.
Statistical analyses Age-specific RIE count was calculated as the total number of RIEs from the day after the previous visit until the end of the day of the following 3 month visit. RIE count for a child during any time period was calculated as a rate per year. The primary aim was to assess RIE rates in relation to risk of islet autoimmunity at different time periods. Associations were examined by time-dependent Cox proportional hazard models. Children were censored after the day of the last negative islet autoimmunity result or the day of the 48 month sample, whichever came first. The 3 month RIE rate on the day of seroconversion was first examined for association with hazard of islet autoimmunity. The date of seroconversion was considered the date of the first positive islet autoimmunity sample. The effect of RIE at the time of seroconversion was assessed using $\mathrm{HR}$ and $95 \% \mathrm{CI}$, as calculated from the time-dependent Cox regression model. The period before seroconversion was defined as the time from date of enrolment up to date of the last 3 month visit prior to the day of seroconversion. The effect of RIEs on subsequent seroconversion was modelled by several time-varying predictors that looked at RIEs in different periods relative to this last visit prior to seroconversion. The RIE rate was examined over the entire period from enrolment to the last visit before seroconversion ('cumulative'), in the first year after enrolment ('early'), and in each year before seroconversion ('recent'). The early RIE rate was modelled as a step function and examined over the entire year and at 3 month intervals within the first year. Recent RIE rates were modelled by a lag function and examined at 3 month intervals up to 2 years prior to seroconversion. All models were adjusted for sex, $H L A-D Q$ genotype, and family history of type 1 diabetes (FDR) by including them as covariates, as well as for country, by fitting a separate baseline hazard. In models examining RIE rates before seroconversion, an adjustment was made for the age of the child at the last visit to ensure that RIE rates were counted over a similar age period.

The second aim was to characterise in a subgroup analysis any significant time-dependent association between RIE rate and islet autoimmunity. Further characterisation included testing by seasonality, febrile symptoms and respiratory infection subcategory. RIE subcategories were included together in multivariate models when possible to identify types that had an independent association with islet autoimmunity. Cox regression models also tested whether RIEs were associated with progression to seroconversion to a second autoantibody type or type 1 diabetes by age 6 years.

All final models were adjusted for SNPs PTPN22 (rs2476601), INS (rs689), ERBB3 (rs2292239), SH2B3 (rs3184505), CLEC16A (rs12708716) and for dietary probiotics before 3 months of age, which have been previously associated with islet autoimmunity in TEDDY [18-20], as well as for potential confounders, including preterm birth, low birthweight, mother's first child, maternal education and 
Table 1 Description of cohort

\begin{tabular}{|c|c|c|c|c|}
\hline \multirow[t]{3}{*}{ Factor } & \multirow{3}{*}{$\begin{array}{l}\text { Islet autoimmunity-positive } \\
\text { children, } n / \text { total }(\%)^{\mathrm{a}}\end{array}$} & \multicolumn{3}{|c|}{ Multivariate proportional hazard model of factors associated with $\mathrm{IA}^{\mathrm{b}}$} \\
\hline & & \multirow{2}{*}{$\begin{array}{l}\text { Unadjusted } \\
\text { HR (95\% CI) }\end{array}$} & \multicolumn{2}{|l|}{ Adjusted $^{\mathrm{c}}$} \\
\hline & & & $\mathrm{HR}(95 \% \mathrm{CI})$ & $p$ value \\
\hline \multicolumn{5}{|l|}{$H L A-D R$ genotype } \\
\hline$D R 4-D Q 8 / D R 4-D Q 8$ & $76 / 1551(4.9)$ & 1.00 & 1.00 & Ref. \\
\hline$D R 3-D Q 2 / D R 4-D Q 8$ & $229 / 3063(7.5)$ & $1.53(1.18,1.98)$ & $1.48(1.13,1.94)$ & 0.004 \\
\hline$D R 4-D Q 8 / D R 8-D Q 4$ & $69 / 1361(5.1)$ & $1.04(0.75,1.45)$ & $1.08(0.77,1.51)$ & 0.90 \\
\hline$D R 3-D Q 2 / D R 3-D Q 2$ & $60 / 1637(3.7)$ & $0.75(0.54,1.06)$ & $0.71(0.50,1.02)$ & 0.06 \\
\hline$D R$ - $D Q$-FDR specific & 20/257 (7.8) & $0.64(0.38,1.09)$ & $0.68(0.40,1.16)$ & 0.16 \\
\hline \multicolumn{5}{|l|}{ Family history of T1D } \\
\hline No & $353 / 6997(5.0)$ & 1.00 & 1.00 & Ref. \\
\hline Yes & 101/872 (11.6) & $2.62(2.04,3.37)$ & $2.42(1.90,3.14)$ & $<0.0001$ \\
\hline \multicolumn{5}{|c|}{ Sex } \\
\hline Male & $251 / 4010(6.3)$ & 1.00 & 1.00 & Ref. \\
\hline Female & $203 / 3859(5.3)$ & $0.82(0.68,0.99)$ & $0.78(0.64,0.94)$ & 0.01 \\
\hline & & Country & & \\
\hline USA & $150 / 3279(4.6)$ & 1.00 & 1.00 & Ref. \\
\hline Finland & $117 / 1717(6.8)$ & $1.45(1.13,1.86)$ & $1.46(1.12,1.92)$ & 0.006 \\
\hline Germany & $36 / 525(6.9)$ & $1.24(0.85,1.80)$ & $1.24(0.82,1.86)$ & 0.13 \\
\hline Sweden & $151 / 2348(6.4)$ & $1.33(1.06,1.66)$ & $1.28(1.00,1.62)$ & 0.04 \\
\hline \multicolumn{5}{|l|}{ rs2476601 (PTPN22-A) } \\
\hline No & $287 / 5467(5.2)$ & & 1.00 & Ref. \\
\hline Yes & $135 / 1426(9.5)$ & & $1.76(1.43,2.16)$ & $<0.0001$ \\
\hline \multicolumn{5}{|l|}{ rs689 (INS-A) } \\
\hline No & $293 / 4073(7.2)$ & & 1.00 & Ref. \\
\hline Yes & $155 / 3374(4.6)$ & & $0.68(0.56,0.83)$ & 0.0002 \\
\hline \multicolumn{5}{|l|}{ rs2292239 (ERBB3-T) } \\
\hline No & $154 / 3151(4.9)$ & & 1.00 & Ref. \\
\hline Yes & $268 / 3743(7.2)$ & & $1.47(1.20,1.79)$ & 0.0002 \\
\hline \multicolumn{5}{|l|}{ rs3184505 (SH2B3-T) } \\
\hline No & $103 / 2138(4.8)$ & & 1.00 & Ref. \\
\hline Yes & $319 / 4756(6.7)$ & & $1.31(1.05,1.64)$ & 0.02 \\
\hline \multicolumn{5}{|l|}{ rs12708716 (CLEC16A-G) } \\
\hline No & $205 / 3042(6.7)$ & & 1.00 & Ref. \\
\hline Yes & $217 / 3836(5.7)$ & & $0.83(0.69,1.00)$ & 0.05 \\
\hline \multicolumn{5}{|c|}{ Dietary probiotics by age 90 days } \\
\hline No & $396 / 6743(5.9)$ & & 1.00 & Ref. \\
\hline Yes & $58 / 1126(5.2)$ & & $0.73(0.54,0.98)$ & 0.04 \\
\hline
\end{tabular}

${ }^{\text {a }}$ All SNP data available for only 6860 of the 7869 children

${ }^{\mathrm{b}}$ Preterm birth (yes $=5.6 \%$ ), low birthweight (yes $=3.4 \%$ ), maternal primary education (yes $=20.0 \%$ ), trade school or some college $($ yes $=25.3 \%)$, graduated college (yes $=54.7 \%$ ), mother's first child (yes $=44.8 \%$ ) and maternal smoking (yes $=12.8 \%$ ) were not associated with hazard of islet autoimmunity ( $p$ values $>0.15$ )

${ }^{c}$ Adjusted for SNPs and dietary probiotics

Ref, reference; T1D, type 1 diabetes

maternal smoking during pregnancy. These additional covariates were only available for children followed to at least 9 months of age. As the timing of RIEs on islet autoimmunity was of interest, statistical tests examining RIE rates with different temporal relationships to islet autoimmunity risk were considered independent and two-sided; $p$ values less than 0.05 
were statistically significant. In the subgroup analysis, $p$ values were also used to help characterise the RIE associations with islet autoimmunity. All statistical analyses were performed using SAS, Version 9.4 (SAS Institute Cary, NC, USA) and GraphPad PRISM 5.03 (GraphPad Software, San Diego, CA, USA) for figures.

\section{Results}

Overall, 454 out of 7869 (5.8\%) of the enrolled children developed persistent islet autoimmunity between 3 and 48 months of age. The incidence (2.0/100 person-years) declined by age (Fig. 1), and differed by sex, country, FDR, $H L A-D Q-D R$ genotype and SNPs (Table 1). Of the 454 children who developed islet autoimmunity, $375(82.6 \%)$ had a single autoantibody at the time of seroconversion, including 155 children (34.1\%) with GADA only (mean age at seroconversion: 26.9 months), 215 (47.4\%) with IAA only (mean age: 20.7 months) and $5(1.1 \%)$ with IA2A only (mean age: 31.4 months). The remaining 79 (17.4\%) children (mean age: 23.0 months) were positive for multiple autoantibodies at seroconversion.

A total of 87,327 RIEs were reported (RIE incidence $=3.9$ / person-year). Age-specific RIE rates were strongly correlated with the decline in islet autoimmunity incidence (Spearman's correlation $=0.71, p=0.003$, Fig. 1). A seasonal trend in RIE rates was consistently observed across age and demographic groups, with the highest and lowest rates seen from October to February and from May to August, respectively. RIE rates according to the month reported were: January $=4.6 /$ personyear, February $=5.0$, March $=4.5$, April $=3.9$, May $=3.4$, June $=2.4$, July $=1.6$, August $=2.9$, September $=4.2$,

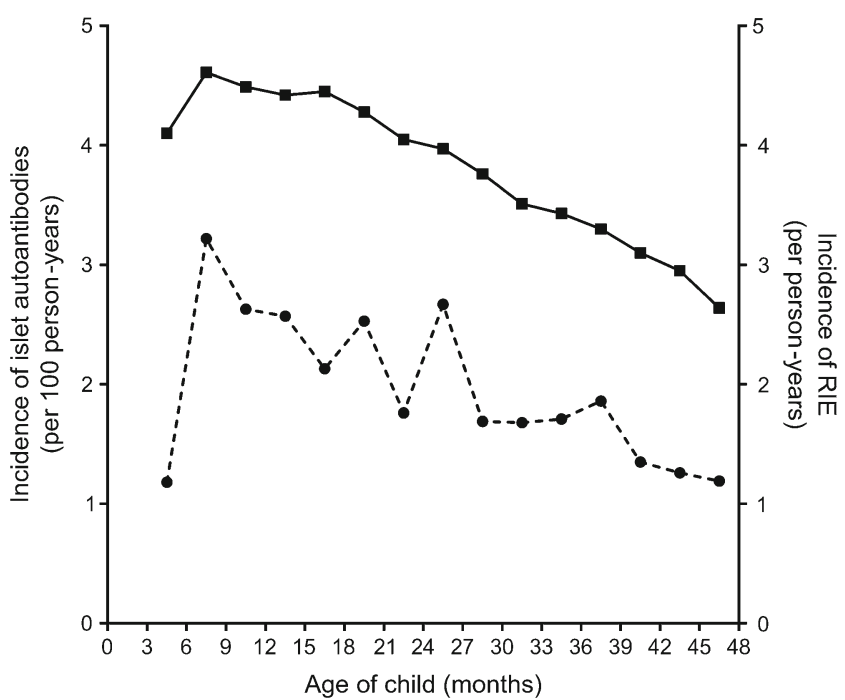

Fig. 1 Incidence of islet autoimmunity per 100 person-years (dashed line) peaked between 6 and 9 months of age, and declined thereafter. Incidence of RIE per person-years (solid line) also peaked between 6 and 9 months and declined thereafter. Age-specific RIE rates were strongly correlated with the decline in islet autoimmunity incidence
October $=4.6$, November $=4.6$, December $=4.8$. The most frequent diagnosis related to RIEs was the common cold, which was reported in $83.2 \%$ of the episodes, followed by bronchitis and lower respiratory tract infection $(23.0 \%)$, middle ear infection $(19.0 \%)$, conjunctivitis $(4.8 \%)$, laryngitis $(2.1 \%)$, tonsillitis or streptococcal pharyngitis $(1.9 \%)$, influenza-like illness (1.2\%), enterovirus-like illness (1.0\%), sinusitis $(0.9 \%)$, respiratory syncytial virus infection $(0.3 \%)$ and other respiratory tract infections grouped together (5.0\%). A fever symptom was reported in $41.1 \%$ of RIEs.

The RIE rate (count/year) at the time of seroconversion was not associated with the risk of islet autoimmunity (HR $1.00[95 \%$ CI 0.98, 1.02]; $p=0.73$ ) (Fig. 2a). However, an increase in the cumulative RIE yearly rate from enrolment to the last visit before seroconversion was significantly associated with islet autoimmunity (HR 1.05 [95\% CI 1.01, 1.08]; $p=0.008$ ). More specifically, an increase in RIE rate within any given 12 month period was associated with subsequent islet autoimmunity seroconversion (HR 1.06 [95\% CI 1.02, 1.09]; $p<0.001$ ). This increase in risk was seen with RIEs at 0 to 3 months, 3 to 6 months and 6 to 9 months before seroconversion (HR [95\% CI]: 1.03 [1.01, 1.06], 1.02 [95\% CI 1.00, 1.05] and 1.03 [1.00, 1.05]; $p$ values $0.003,0.06$ and 0.05 , respectively), but not prior to 9 months (HR 1.01 [95\% CI 0.98, 1.04]; $p=0.41$ ) (Fig. 2a). RIEs during the first year after enrolment were not associated with islet autoimmunity (HR 1.00 [95\% CI 0.97, 1.03]; $p=0.99$ ). There was no marked difference between the effect sizes described above for islet autoimmunity and effect sizes observed for seroconversion with IAA or GADA as the first-appearing antibody (data not shown).

For each $1 /$ year rate increase in the number of respiratory infections occurring in a 9 month period, the hazard of islet autoimmunity increased significantly by $5.6 \%$ (95\% CI $2.5 \%$, $8.8 \%), p<0.001$. There were no interactions by HLA, sex, family history of diabetes (data not shown) or age of child (ESM Fig. 1). The percentage change in hazard was also similar in Finland $+7.1 \%(95 \% \mathrm{CI}+1.7 \%,+12.7 \%)$, Germany $+14.3 \%(95 \% \mathrm{CI}+5.2 \%,+24.2 \%)$, Georgia/Florida $+10.3 \%$ $(95 \% \mathrm{CI}+0.0 \%,+21.1 \%)$ and Washington state $+7.8 \%(95 \%$ CI $-2.7 \%,+19.3 \%)$. In Sweden, an increasing hazard was seen in children older than 24 months $+7.6 \%$ (95\% CI $-0.5 \%,+16.3 \%)$, but not in younger children $-2.9 \%(95 \%$ CI $-10.4 \%,+5.1 \%)$. No association was seen in Colorado $-3.6 \%$ (95\% CI $-13.0 \%,+6.8 \%)$. Compared with the lower quartile RIE count in the 0 to 9 month period ( $<2$ per year), a $9.4 \%$ increased risk of islet autoimmunity within the next 3 months was observed if RIEs were within the interquartile range (RIE count 2-5/year; HR 1.09 [95\% CI 0.86, 1.39]; $p=0.47$ ), and a $46.6 \%$ increased risk of islet autoimmunity if RIEs were in the upper quartile (RIE count $>5 /$ year; HR 1.5 [95\% CI 1.12, 1.93]; $p=0.006$ ).

This risk association depended on the season of an RIE and was linked to RIEs occurring during the winter months of 


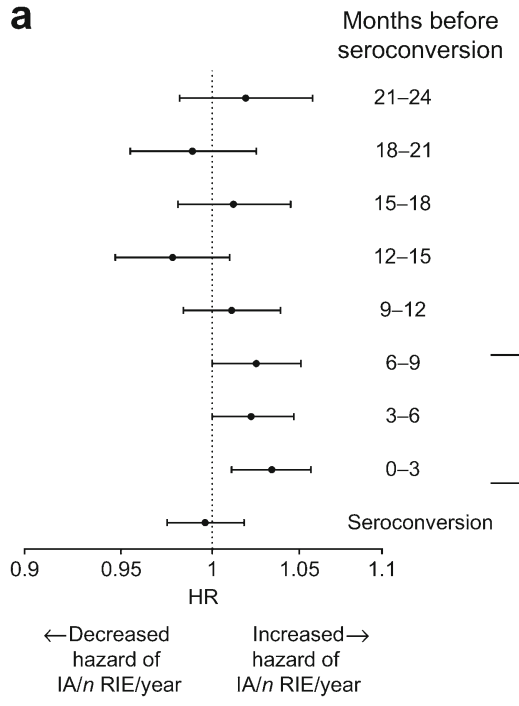

b

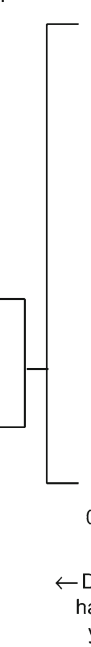

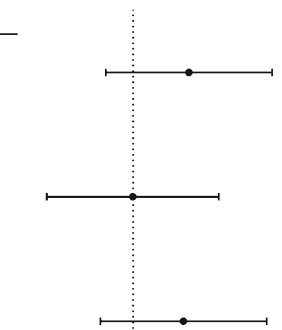

3 Month season

C

Spring

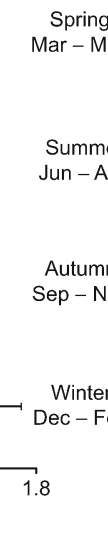

Increased $\rightarrow$
hazard of IA
yes vs no
-
-

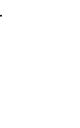

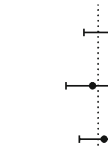

pes of RIE reported in the winter

Conjunctivitis

Bronchitis and lower respiratory tract infections Infection of middle ear and mastoid process Sinusitis

Tonsillitis or streptococcal pharyngitis

Laryngitis and tracheitis

Enterovirus-like illness

Influenza-like illness

Common cold

\begin{tabular}{lllllllll}
\hline 0.2 & 0.4 & 0.7 & 1 & 1.5 & 2.5 & 4.5 & 7.0 & 12
\end{tabular}

HR
Fig. 2 (a) RIEs occurring between 0 and 3 months, 3 and 6 months, and 6 and 9 months before the autoantibody seroconversion period were associated with increased risk of islet autoimmunity ( $p \leq 0.05$ for all), whereas RIE rates in the other 3 month periods were not $(p>0.10)$. (b) In a multivariate analysis, the presence of a winter but not a spring, summer or autumn RIE, occurring in the 9 month period before seroconversion, was associated with islet autoimmunity. (c) In a multivariate analysis, the specific infections in the winter with significant association with islet autoimmunity were sinusitis, laryngitis and tracheitis, influenza-like illness and common cold
December, January and February (Fig. 2b). Risk of islet autoimmunity increased by $42.4 \%$ (yes vs no; HR 1.42, [95\% CI $1.16,1.74] ; p<0.001$ ) when a winter RIE was reported (yes vs no), while no association was seen for RIEs reported in other seasons. Both febrile and non-febrile RIEs were associated with an increased risk of islet autoimmunity (HR 1.07 [95\% CI 1.02, 1.12], $p=0.005$; HR 1.05 [95\% CI 1.01, 1.09], $p=0.02$, respectively); however, febrile RIEs showed a more consistent association when analysed in different age groups (ESM Fig. 1). Of the individual subcategory infections recorded during the winter months, common cold, influenza-like illnesses, sinusitis, and laryngitis and tracheitis were independently associated with islet autoimmunity (Fig. 2c). In other seasons, none of the subcategories of RIE was associated with the risk of islet autoimmunity (Table 2).

The islet autoimmunity risk characterisation by RIE 09 months before islet autoimmunity seroconversion was not associated with number of islet autoantibodies or rate of progression from islet autoimmunity to type 1 diabetes (data not shown). The characterisation was also not explained by the remaining factors significantly associated with islet autoimmunity (Table 1) and available for $86 \%$ of the participants (6800/7869 participants; RIE 0-9 month $n$ /year on risk of islet autoimmunity; HR 1.05 [95\% CI 1.02, 1.09]; $p=0.001$ ).

\section{Discussion}

This prospective analysis showed that among young children, the number of RIEs within any 9 month period was associated with the subsequent onset of islet autoimmunity within the following 3 months. It is unlikely this finding was due to generally increased susceptibility to infections in the seroconverting children, as the association with risk of islet autoimmunity was only observed for RIEs prior to the last autoantibody-negative sample and not for RIEs during the period of islet autoimmunity seroconversion. Furthermore, characterisation of the RIEs in the 9 month period revealed that the common cold, influenza-like illness, sinusitis and laryngitis/tracheitis, were the types of respiratory infections in the winter that were independently associated with autoimmunity. Further pathogen-specific studies should focus on this particular period in great detail to determine the causative microbes.

The magnitude of the association between RIEs within any 9 month period and the risk of islet autoimmunity was moderate: the median RIE rate was 4 RIEs/year and ranged from $0 /$ year at the 10 th percentile to $8 /$ year at the 90 th percentile, with the hazard of islet autoimmunity increasing by $5.6 \%$ with each 1 year rate increase in respiratory infection rate. While the rate showed a monotonically increasing impact on islet autoimmunity hazard, a more pronounced effect on hazard of islet autoimmunity (46.6\%) was observed when RIEs occurred at the highest rate (upper quartile rate $>5 /$ year). This association indicates that RIEs are not a good predictor of whether an individual child will seroconvert for islet autoantibodies. However, at a population level, comparing groups with different RIE rates, the observed association is relatively consistent by country, remaining when adjusting for many other risk factors, and the association is in line with previous 


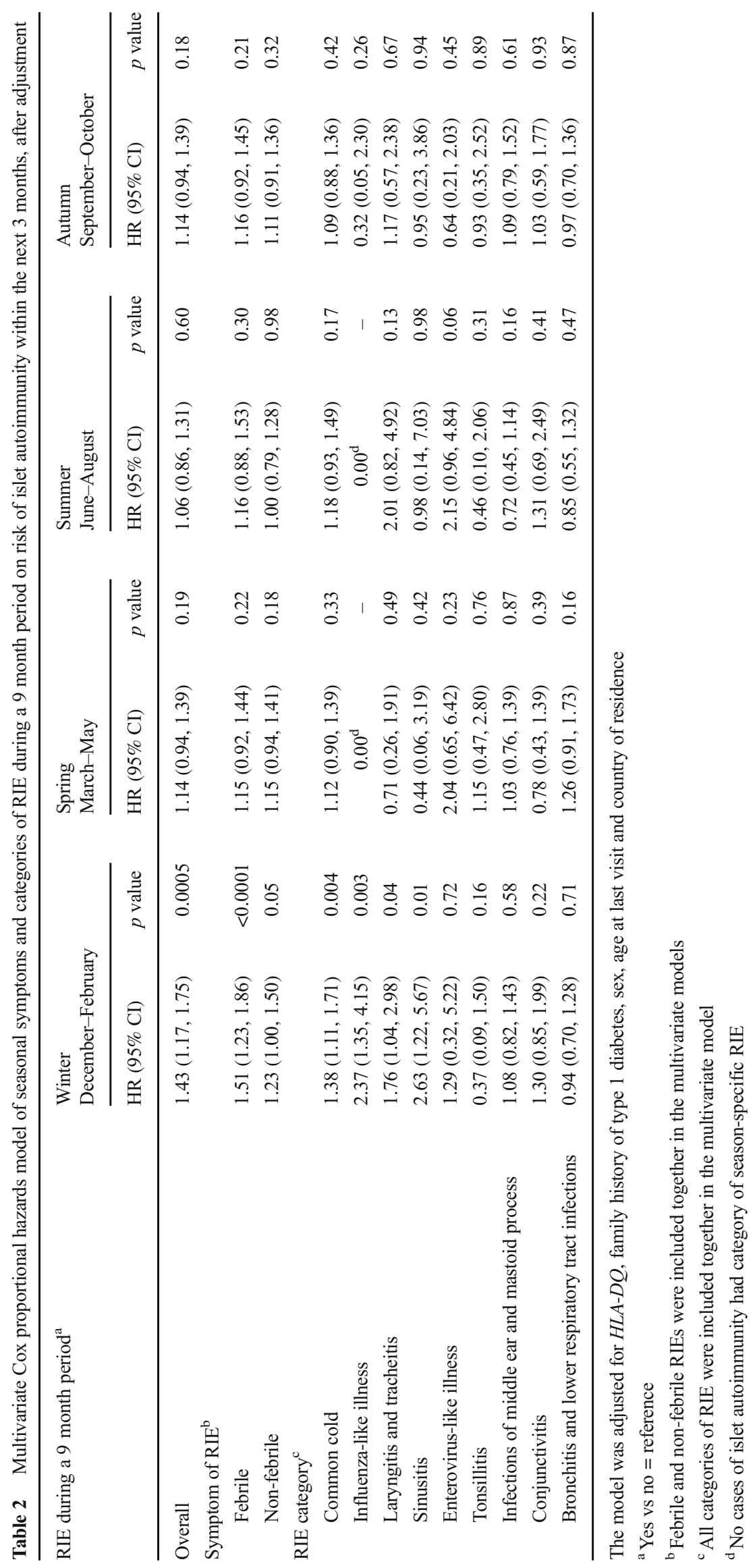


findings on type 1 diabetes [1, 4-7], which suggests a biological mechanism is probably present. Further research is required to understand whether the moderate effect of RIE on islet autoimmunity is due to the role of specific microbes or interactions with other unknown risk factors. The firstappearing autoantibody (IAA vs GADA) has been suggested to represent two different phenotypes of autoimmunity [20, 21]. In the present study, the effect size did not markedly differ between seroconversion with IAA or GADA as the firstappearing antibody, which suggests that RIEs may trigger both autoimmunity pathways.

The RIE-associated increased risk of islet autoimmunity was observed year-round, but it was observed primarily for winter RIEs (Fig. 2b), which was the high season for respiratory infections in all TEDDY sites. These specific infection types observed in the winter season (Fig. 2c) are most often caused by viruses such as rhino-, corona-, influenza-, respiratory syncytial, parainfluenza-, adeno-, entero- and metapneumoviruses [22-24]. In sinusitis, bacteria such as Moraxella catarrhalis, Streptococcus pneumoniae and Haemophilus influenzae are also frequently involved [25]. A pronounced increase in the risk of islet autoimmunity was observed only when RIEs occurred at a high rate. This may imply involvement of more than one microbe in the initiation of autoimmunity; however, our findings do not identify the particular culprit microbe(s). Although the marked differences in islet autoimmunity risk associated with various anatomical sites of RIE shown in Fig. 2c (e.g. infection located in conjunctiva [conjunctivitis] vs infection in the sinuses [sinusitits]) raise the possibility of a more specific or limited set of pathogens, it should also be considered that a generally high rate of infection in the 9 months preceding islet autoimmunity seroconversion may play a role. The latter scenario could be consistent with the 'fertile field' hypothesis [26], suggesting that many infections could induce a temporary proinflammatory cytokine milieu (a fertile field), promoting activation of autoreactive $\mathrm{T}$ cells.

Enteroviruses have been suggested to trigger islet autoimmunity [7, 27] and detection of enterovirus RNA in blood has been shown to increase islet autoimmunity risk in the following 6 months [8], resembling the temporal pattern observed for RIEs in the present study. Nevertheless, in the present study, no association was found between the subcategory of enterovirus-like illnesses and islet autoimmunity risk. This is probably because of the low sensitivity for identifying these infections from parental reports. Enterovirus infections are mostly subclinical, and clinically manifest most often with fever or non-specific upper respiratory tract symptoms, such as in the common cold [28]. Only some enterovirus infections can be recognised based on clinical representation, such as hand, foot and mouth disease and herpangina. Therefore, we suspect that in the present study, only a minor fraction of enterovirus infections has been classified as enterovirus-like illnesses, and most have been diagnosed as common colds.

Febrile RIEs showed a more consistent risk association with islet autoimmunity compared with non-febrile RIEs, suggesting that fever may be a factor increasing islet autoimmunity risk within RIEs. The clinical course of febrile infections tends to be more severe and is often associated with pronounced viraemia that allows the spread of the virus to the pancreas. High severity of infections may also be linked with more intense beta cell stress: acute infections induce stress in beta cell endoplasmic reticulum because of insulin resistance and increased insulin excretion [29]. The stress reaction in endoplasmic reticulum can cause post-translational modifications in beta cell autoantigens [30-33], making them more immunogenic and thus promoting autoimmunisation. Accordingly, RIE-induced beta cell stress paving the way for autoimmunisation is one plausible explanation for the observed association between RIEs and increased islet autoimmunity risk.

Serum samples used to test for islet autoantibodies and TEDDY diary book infection records were collected at each visit at regular 3 month intervals. Visit compliance was relatively high (87\%); if families missed a visit, parents were asked to recall all infections since the last visit. The temporal associations of RIE with islet autoimmunity were also examined among individuals with higher compliance, up to perfect compliance, and the magnitude of the associations remained the same. In the present study, all participants reporting infections were still at risk for islet autoimmunity. Therefore, a possible increase in vigilance and reporting of infections after parents have been informed about their child's islet autoimmunity was not an issue in this study.

We also controlled for the possibility of overestimation of infection rate because of multiple illness and symptom reports during a single infection by binding together reports into infectious episodes that originated from the same infection (for details see Lönnrot et al [16]).

Certain study limitations remained. Selection bias is likely as age of mother, country, education and other factors were associated with dropout rate in the TEDDY study [34, 35]. Misclassification of infections, as discussed for enterovirus infections, was an important limitation. Many respiratory infections have quite similar symptoms, and caution is needed in interpreting the results based on infection subcategories. Another limitation of the study is capturing only clinically overt infections. These are generally regarded as the mere tip of the iceberg of microbial exposures, which may all potentially influence islet autoimmunity risk. It is also possible that some conditions classified as infections were actually noninfectious. For example, infectious and allergic rhinitis can exhibit fairly similar symptoms. However, febrile RIEs showed a more consistent risk associated with islet autoimmunity compared with non-febrile RIEs, suggesting that this association is truly related to infections. 
Conclusions The present prospective study consisting of almost 8000 participants has confirmed the findings of two smaller prospective studies (the Norwegian MIDIA study with 885 participants and German BABYDIET study with 148 participants $[4,5])$ of the risk association between respiratory infection and islet autoimmunity. The observed temporal pattern suggests an islet autoimmunity-triggering effect by respiratory infections. Next, effort will be made to identify the specific microbes, probably viruses, by analysing biological samples taken from TEDDY participants during the 0-9 month time window prior to the development of islet autoimmunity.

Acknowledgements TEDDY families are warmly acknowledged for their participation in this study. The TEDDY Study Group is acknowledged for excellent collaboration (see ESM).

Data availability The data that support the findings of this study are available from the National Institute of Diabetes and Digestive and Kidney Diseases (NIDDK) Central Repository on request.

Funding This study was funded by U01 DK63829, U01 DK63861, U01 DK63821, U01 DK63865, U01 DK63863, U01 DK63836, U01 DK63790, UC4 DK63829, UC4 DK63861, UC4 DK63821, UC4 DK63865, UC4 DK63863, UC4 DK63836, UC4 DK95300, UC4 DK100238, UC4 DK106955 and contract number HHSN267200700014C from the NIDDK, National Institute of Allergy and Infectious Diseases (NIAID), National Institute of Child Health and Human Development (NICHD), National Institute of Environmental Health Sciences (NIEHS), JDRF and Centers for Disease Control and Prevention (CDC). This work supported in part by National Institutes of Health (NIH)/National Center for Advancing Translational Sciences (NCATS) Clinical and Translational Science Awards to the University of Florida (UL1 TR000064), the University of Colorado (UL1 TR001082) and Sigrid Juselius Foundation (grant to HH).

Duality of interest The authors declare that there is no duality of interest associated with this manuscript.

Contribution statement All authors attest to meeting ICMJE uniform requirements for authorship by making substantial contributions to the conception and design of this study, the acquisition, analysis and interpretation of the data and to drafting or revising the article for intellectual content. All authors have approved the final version of the article to be published. ML and KL are responsible for the integrity of the work as a whole.

\section{References}

1. Op de Beeck A, Eizirik DL (2016) Viral infections in type 1 diabetes mellitus-why the beta-cells? Nat Rev Endocrinol 12:263-273

2. Bach JF, Chatenoud L (2012) The hygiene hypothesis: an explanation for the increased frequency of insulin-dependent diabetes. Cold Spring Harb Perspect Med 4:a00779

3. Snell-Bergeon JK, Smith J, Dong F et al (2012) Early childhood infections and the risk of islet autoimmunity. The Diabetes Autoimmunity Study in the Young (DAISY). Diabetes Care 35: 2553-2558

4. Rasmussen T, Witsø E, Tapia G, Stene LC, Rønningen KS (2011) Self-reported lower respiratory tract infections and development of islet autoimmunity in children with the type 1 diabetes high-risk HLA genotype: the MIDIA study. Diabetes Metab Res Rev 27: 834-837

5. Beyerlein A, Wehweck F, Ziegler AG, Pflueger M (2013) Respiratory infections in early life and the development of islet autoimmunity in children at increased type 1 diabetes risk evidence from the BABYDIET Study. JAMA Pediatr 167:800-807

6. Beyerlein A, Donnachie E, Jergens S, Ziegler AG (2016) Infections in early life and development of type 1 diabetes. JAMA 319:1899

7. Yeung WC, Rawlinson WD, Craig ME (2011) Enterovirus infection and type 1 diabetes mellitus: systematic review and metaanalysis of observational molecular studies. BMJ 342:d35

8. Oikarinen S, Martiskainen M, Tauriainen S et al (2011) Enterovirus RNA in blood is linked to the development of type 1 diabetes. Diabetes 60:276-279

9. Stene LC, Oikarinen S, Hyöty H et al (2010) Enterovirus infection and progression from islet autoimmunity to type 1 diabetes. The Diabetes and Autoimmunity Study in the Young (DAISY). Diabetes 59:3174-3180

10. Krogvold L, Edwin B, Buanes T et al (2015) Detection of a lowgrade enteroviral infection in the islets of langerhans of living patients newly diagnosed with type 1 diabetes. Diabetes 64:16821687

11. Richardson SJ, Willcox A, Bone AJ, Foulis AK, Morgan NG (2009) The prevalence of enteroviral capsid protein vp1 immunostaining in pancreatic islets in human type 1 diabetes. Diabetologia 52:1143-1151

12. TEDDY Study Group (2007) The Environmental Determinants of Diabetes in the Young (TEDDY) study: study design. Pediatr Diabetes 8:286-298

13. TEDDY Study Group (2008) The Environmental Determinants of Diabetes in the Young (TEDDY) study. Ann N Y Acad Sci 1150:113

14. Bonifacio E, Yu L, Williams AK et al (2010) Harmonization of glutamic acid decarboxylase and islet antigen-2 autoantibody assays for national institute of diabetes and digestive and kidney diseases consortia. J Clin Endocrinol Metab 95:3360-3367

15. American Diabetes Association (2016) Standards of Medical Care in Diabetes-2016. Diabetes Care 39:S1-S112

16. Lönnrot M, Lynch K, Elding Larsson H et al (2015) A method for reporting and classifying acute infectious diseases in a prospective study of young children: TEDDY. BMC Pediatr 15:24

17. Chawla R, Kellner JD, Astle WF (2001) Acute infectious conjunctivitis in childhood. Paediatr Child Health 6:329-335

18. Törn C, Hadley D, Lee HS et al (2015) Role of type 1 diabetesassociated SNPs on risk of autoantibody positivity in the TEDDY study. Diabetes 64:1818-1829

19. Uusitalo U, Liu X, Yang J et al (2016) Association of early exposure of probiotics and islet autoimmunity in the TEDDY study. JAMA Pediatr 170:20-28

20. Krisher JP, Lynch KF, Schatz DA et al (2013) The 6 year incidence of diabetes-associated autoantibodies in genetically at-risk children: the TEDDY study. Diabetologia 58:980-987

21. Ilonen J, Hammais A, Laine AP et al (2013) Patterns of B-cell autoantibody appearance and genetic associations during the first years of life. Diabetes 62:3636-3640

22. Heikkinen T, Järvinen A (2003) The common cold. Lancet 361:5159

23. Mäkelä MJ, Puhakka T, Ruuskanen O et al (1998) Viruses and bacteria in the etiology of the common cold. J Clin Microbiol 32: 539-542

24. Miller KE, Gebretsadik T, Carroll KN et al (2013) Viral etiologies of infant bronchiolitis, croup and upper respiratory illness during 4 consecutive years. Pediatr Infect Dis J 32:950-955

25. Nocon CC, Baroody FM (2014) Acute rhinosinusitis in children. Curr Allergy Asthma Rep 14:443 
26. von Herrath MG, Fujinami RS, Whitton JL (2003) Microorganisms and autoimmunity: making the barren field fertile? Nat Rev Microbiol 1:151-157

27. Morgan NG, Richardson SJ (2014) Enteroviruses as causative agents in type 1 diabetes: loose ends or lost cause? Trends Endocrinol Metab 25:611-619

28. Pallansch MA, Roos RP (2001) Enteroviruses: polioviruses, coxsackieviruses, echoviruses and newer enteroviruses. In: Fields BN, Knipe DM, Howley PM, Griffin DE (eds) Field's virology. Lippincot, Williams and Wilkins, Philadelphia, pp 723-775

29. Yki-Järvinen H, Sammalkorpi K, Koivisto VA, Nikkilä EA (1989) Severity, duration and mechanisms of insulin resistance during acute infections. J Clin Endocrinol Metab 69:317-323

30. Dunne JL, Overbergh L, Purcell AW, Mathieu C (2012) Posttranslational modifications of proteins in type 1 diabetes: the next step in finding the cure? Diabetes 61:1907-1914
31. van Lummel M, Duinkerken G, van Veelen PA et al (2014) Posttranslational modification of HLA-DQ binding islet autoantigens in type 1 diabetes. Diabetes 63:237-247

32. McGinty JW, Chow IT, Greenbaum C et al (2014) Recognition of posttranslationally modified GAD65 epitopes in subjects with type 1 diabetes. Diabetes 63:3033-3040

33. Clark AL, Urano F (2016) Endoplasmic reticulum stress in beta cells and autoimmune diabetes. Curr Opin Immunol 43:60-66

34. Bennett Johnson S, Lynch K, Baxter J et al (2016) Predicting later study withdrawal in participants active in a longitudinal birth cohort study for 1 year: the TEDDY study. J Pediatr Psychol 41:373-383

35. Bennett Johnson S, Lee HS, Baxter J et al (2011) The Environmental Determinants of Diabetes in the Young (TEDDY) study: predictors of early study withdrawal among participants with no family history of type 1 diabetes. Pediatr Diabetes 12: 165-171 